\title{
Hepatosplenic T cell lymphoma in a patient treated with infliximab for crohn's disease not responding to conventional therapies
}

\begin{abstract}
Crohn's disease is a type of inflammatory bowel disease implying the immune system. The disease by itself might evolve in deteriorating symptoms that are overwhelming for the patient, such as generalized enteritis, which cannot be easily put into remission. In addition, a correlation between Crohn's disease and lymphoma presentation, especially Hepatosplenic-T cell lymphoma, has been reported in young males. Symptoms of the disease can be controlled with mild medication such as amino salicylates or corticosteroids while persisting symptoms or recurrent episodes can be furthely managed with immune modulators such as azathioprine, methotrexate or antiTNF monoclonal antibodies like infliximab. A case of a patient with Crohn's disease, resistant to conventional immunosuppressive therapies, including azathioprine, presenting lymphoma while on anti-TNF therapy is reported.
\end{abstract}

Volume I Issue I - 2015

Komitopoulou Anna, Delimpasi Sossana, Karakasis Demetrios, Harhalakis Nikolaos Department of Hematology and Bone Marrow Transplantation Unit, Evangelismos Hospital, Greece

Correspondence: Komitopoulou Anna, Haematologist, Department of Hematology and Bone Marrow Transplantation Unit, Evangelismos Hospital, 45-47 Ipsilantou Str,Athens, Greece,Email annakom@yahoo.com

Received: February 04, 2015 | Published: March 10, 2015

Keywords: crohn's disease, lymphoma, immunomodulators, immunosuppression, hepatosplenic T-lymphoma

\section{Introduction}

Hepatosplenic T-cell Lymphoma (HSTCL) is an extranodal and systematic neoplasm derived from cytotoxic T-cells usually of $\gamma \delta$ T-cell receptor type. HSTCL is a rare lymphoma $(<1 \%$ of all non Hodgkin's lymphomas-NHL) which origin has been correlated with chronic immune suppression. Patients with Crohn's disease, a condition involving autoimmunity, present with an increased risk of lymphoma. ${ }^{1}$ Furthermore, rare cases of Hepatosplenic T-cell lymphomas have been reported in adolescent and young adult patients with Crohn's disease treated with infliximab. ${ }^{2}$ Infliximab is a chimeric IgG monoclonal antibody known as a biological response modifier directed against tumor necrosis factor-a (TNF- $\alpha)$, a cytokine which plays an important role in the inflammation process.

The case of a 33-years old man diagnosed in 1997 with Crohn's disease, by radiography and histology of mucosal biopsy taken at colonoscopy, is reported. At diagnosis, the patient was initially treated with steroids and one year later azathioprine therapy was introduced due to symptoms evolution. However, the patient had poor compliance to the therapeutic protocol for a long period of time (from 1998 until 2005) and further required dilatation due to intestinal obstruction (2000) and colectomy, as well (2004). From 2005 to May 2008 he was on regular azathioprine therapy. Nevertheless, Crohn's disease remained active despite therapy, and infliximab was initiated in May 2008. During a six months period the patient received a total of five doses of $5 \mathrm{mg} / \mathrm{kg}$ of infliximab intravenously, under concomitant treatment with azathioprine.

After the $5^{\text {th }}$ infusion of infliximab (November 2008) he presented with pyrexia, mainly during afternoon, and fatigue. The patient was admitted to the hospital for further investigation. On physical examination, no specific findings other than hepatosplenomegaly were found. Full blood examination did not reveal any abnormal finding. Biochemical analysis revealed abnormal liver functions: aspartate aminotransferase: 522IU/L (normal values: 5-37IU/
L), alanine aminotransferase: 690IU/L (5-40IU/L), $\gamma$-glutamyl transferase: 201IU/L (7-49IU/L), alkaline phosphatase: 453IU/L (35-129IU/L), lactate dehydrogenase: 1615IU/L (<255IU/L). Regarding coagulation parameters, prothrombin time was $14.5 \mathrm{sec}$ (normal range 11-14sec), International Normalizing Ratio: 1.15, activated Partial Thromboplastin Time: $34.4 \mathrm{sec}$ (normal range: 20$40 \mathrm{sec}$ ). Inflammatory markers such as $\mathrm{C}$ reactive protein levels or erythrocyte sedimentation rate were within the normal range. No pathogens were found in blood cultures and active viral infection was not demonstrated. Abdominal computed tomography confirmed splenomegaly and hepatomegaly $(15 \mathrm{~cm}$ and $19 \mathrm{~cm}$, respectively). No pathologic enlargement of lymph nodes was detected.

Microscopic examination of the liver core biopsy revealed T-Hepatosplenic Lymphoma which was further confirmed with bone marrow biopsy. Specifically, bone marrow biopsy showed infiltration of the marrow with lymphocytes having immunohistochemical characteristics: $\mathrm{CD} 3(+), \mathrm{CD}(2)+, \mathrm{CD} 5(+-), \mathrm{CD} 4(-), \mathrm{CD} 8(+-)$. The percentage of these pathological lymphocytes was $40 \%$. No blastic changes were noted. Immunophenotypic analysis of the marrow sample also showed that the amount of $\mathrm{CD} 3(+)$, TCR $\gamma \delta$ was about $70 \%$ of the pathological lymphocytes, a finding consistent with Hepatosplenic T-cell lymphoma (HTCL). Patient's karyotype analysis was normal (46XY). Due to progressive disease despite initial chemotherapy (cyclophosphamide, adriamycin, vincristine, prednisolone), a salvage regimen was initiated and the patient underwent an allogeneic transplantation from a matched unrelated donor but he finally died because of complications related to transplantation.

We describe the case of a patient with Crohn's disease who had been treated with immunodulatory drug, such as infliximab. Infliximab has shown great efficacy in the treatment of moderate to severe Crohn's disease by binding and blocking the effects of TNF-alpha, a chemokine messenger involved in the autoimmune process. ${ }^{3} \mathrm{TNF}$-alpha enhances inflammatory cytokines, such as interleukins and facilitates leukocyte migration in tissues. Additionally, it is thought to provide protection 
against cancer and infections, mainly via influencing apoptosis and cell survival. ${ }^{4,5}$ Taking the above mechanisms of action of TNF-alpha into consideration, several reports in the literature have correlated the block of TNF-alpha through anti-TNF biological agents, with cancers and lymphomas, especially hepatosplenic lymphoma, in patients with Crohn's disease. Actually, the risk of cancer development is increased three-fold in patients taking anti-TNF therapy compared to the control group. ${ }^{6-8}$ Most of these cases have occurred in patients on concomitant treatment with azathioprine or 6-mercaptopurine. ${ }^{7}$ On the other hand HSTCL occurring in Crohn's disease patients receiving mutagens such as azathioprine or 6-mercaptopurine without taking infliximab is also reported. This fact indicates that causal association between infliximab and HSTCL has not been clearly established. ${ }^{9-12}$

Additionally, the relative risk of lymphomas and other cancers in patients with Crohn's disease is increased. ${ }^{1}$ The patients with Crohn's disease and other autoimmune diseases have a predisposition of developing lymphomas due to their underlying basic intrinsic disease. ${ }^{13-15}$ Lymphoma presentation can occur from weeks to months after infliximab administration, as reported in our patient. ${ }^{16}$ All the above indicates that the issue of association of Crohn's disease, especially in patients taking various immunosuppressive therapies other than biological agents and lymphoma presentation while on infliximab distribution in patients with moderate or severe disease is complicated. Immunity of these patients is altered due to the combination of all the reasons referred. Nevertheless, HSTCL which is a particular type of NHL and the use of infliximab in patients with Crohn's disease is more closely correlated. ${ }^{15-19}$ Anti-TNF therapy is indicated in resistant patients, so as to enhance remission of symptoms and therefore may improve their quality of life. So, more surveillance may be warranted by clinicians in order to reach safe conclusions and keep these innovative drugs, such as biological therapies in the therapeutic armamentarium while avoiding aggressive deteriorations like hepatosplenic lymphoma presentation, mainly occurring in young patients.

\section{Acknowledgements}

None.

\section{Conflict of interest}

The author declares no conflict of interest.

\section{References}

1. Von Roon AC, Reese G, Teare J, et al. The risk of cancer in patients with Crohn's disease. Dis Colon Rectum. 2007;50(6):839-855.

2. Health Canada endorsed important safety information on remicade; 2006.

3. Osterman MT, Lichtenstein GR. Current and future anti-TNF therapy for inflammatory bowel disease. Curr Treat Options Gastroenterol. 2007;10(3):195-207.

4. Goeddel, DV. Connection map for tumor necrosis factor pathway" Science STKE.
5. Chen W, Li N, Chen T, et al. "The lysosome-associated apoptosis-inducing protein containing the pleckstrin homology $(\mathrm{PH})$ and FYVE domains (LAPF), representative of a novel family of PH and FYVE domaincontaining proteins, induces caspase-independent apoptosis via the lysosomal-mitochondrial pathway". J Biol Chem. 2005;280(49):4098540995.

6. Drini M, Prichard PJ, Brown GJ, et al. Hepatosplenic T-cell lymphoma following infliximab therapy for Crohn's disease. Med $J$ Aust. 2008;89(8):464-465.

7. Mackey AC, Green L, Liang LC, et al. Hepatosplenic T cell lymphoma associated with infliximab use in young patients treated for inflammatory bowel disease. J Pediatr Gastroenterol Nutr. 2007;44(2):265-267.

8. Rosh JR, Gross T, Mamula P, et al. Hepatosplenic T-cell lymphoma in adolescents and young adults with Crohn's disease:a cautionary tale? Inflamm Bowel Dis. 2007;13(8):1024-1030.

9. Bongartz T, Sutton AJ, Sweeting MJ, et al. Anti-TNF antibody therapy in rheumatoid arthritis and the risk of serious infections and malignancies: systematic review and meta-analysis of rare harmful effects in randomized controlled trials. JAMA. 2006;295(19):2275-2285.

10. Mittal MS, Milner BJ, Johnston PW, et al. A case of hepatosplenic $\gamma \delta$ T-cell lymphoma with a transient response to fludarabine and alemtuzumab. Eur J Haematol. 2006;76:531-534.

11. Navarro JT, Ribera JM, Mate JL, et al. Hepatosplenic T-gammadelta lymphoma in a patient with Crohn's disease treated with azathioprine. Leuk Lymphoma. 2003;44(3):531-533.

12. Lemann M, de la Valussiere F, Vouhnik Y, et al. Intravenous cyclosporine for refractory attacks of Crohn's disease (CD): long-term follow-up of patients [abstract]. Gastroenterology. 1998;114(number 4, part 2):41784178.

13. Aithal GP, Mansfield JC. Review article:The risk of lymphoma associated with inflammatory bowel disease and immunosuppresing treatment. Aliment Pharmacol Ther. 2001;5(8):1101-1108.

14. Farrell RJ, Ang Y, Kileen P, et al. Increased incidence of NHL in inflammatory bowel disease patientw on immunosuppressive therapy but overall risk is low. Gut. 2000;47(4):514-519.

15. Kong SC, James G. Crohn's disease associated lymphoma after treatment with infliximab. GME Gastroenterology Hepatology and Nutrition. 4(3):100-101.

16. Bickston SJ, Lichtenstein GR, Arseneau KO, et al. The relationship between infliximab treatment and lymphoma in Crohn's disease. Gastroenterology. 1999;117(6):1433-1437

17. Deepak P, Sifuentes H, Sherid M, et al. T-cell non-Hodgkin's lymphomas reported to the FDA AERS with tumor necrosis factor-alpha (TNF- $\alpha$ ) inhibitors: results of the REFURBISH study. Am $J$ Gastroenterol. 2013;108(1):99-105.

18. Ye BD. Inflammatory bowel disease and lymphoproliferative disorders. Korean J Gastroenterol. 2011;58(4):171-177.

19. Scott FI, Vajravelu RK2, Bewtra M3, et al. The benefit-to-risk balance of combining infliximab with azathioprine varies with age: a markov model. Clin Gasrtroenterol Hepatol. 2014;S1542-S3565(14):01143-01144. 